\title{
¿Es el desarrollo de un proyecto una buena herramienta para aprender los principios fundamentales de una materia?
}

\section{Is the development of a project a good tool to learn the main principles of a subject?}

\author{
Marta Pazos, Emilio Rosales, Mª́ngeles Sanromán \\ mcurras@uvigo.es, emiliorv@uvigo.es, sanroman@uvigo.es \\ Ingeniería Química \\ Universidad de Vigo \\ Vigo, España
}

\begin{abstract}
Resumen- Hoy en día, se requiere el uso de herramientas efectivas que refuercen el aprendizaje de los estudiantes. Un ejemplo claro es el uso de Aprendizaje basado en el desarrollo de un proyecto a escala real (ABDPER). ABDPER implica un estudio en profundidad de una situación particular y la utilización de los conceptos adquiridos durante el año académico. La principal ventaja de la enseñanza con ABDPER es que los estudiantes participan activamente en la comprensión de los principios al abstraerse de la teoría enseñada a través del razonamiento inductivo. En nuestro caso, ABDPER se ha aplicado a diversas materias, si bien en este trabajo se expondrá los resultados obtenidos en la materia Reactores y Biotecnología. Para esta propuesta, los estudiantes aplican el conocimiento gradual adquirido durante el semestre al diseño y escalado de una planta de producción industrial e implementarán en el desarrollo del mismo los avances de $\mathrm{I}+\mathrm{D}$ y las Mejores Tecnologías Disponibles (MTD). La respuesta de los estudiantes ha sido muy positiva a esta nueva metodología y se puede concluir que el uso de esta tecnología basada en artículos de investigación científica aumentó las habilidades de los estudiantes en el marco de la asignatura.
\end{abstract}

Palabras clave: Aprendizaje cooperativo, Integración, I+D, Proyecto a Escala Real

Abstract- Nowadays, the use of effective tools that reinforce the students learning is required. A clear example is the use of Learning Based on the Development of a Real Scale Project (LBDRSP). LBDRSP involves an in depth study of a particular situation and the utilization of the concepts acquired during the academic year. The major advantage of teaching using LBDRSP is that the students are actively engaged in figuring out the principles by abstracting from the taught theory through inductive reasoning. In our case, LBDRSP has been applied to different subjects, however in this report, the authors only present the obtained results in the subject Reactors and Biotechnology. The students apply the gradual knowledge acquired during the semester to the design and scale-up of industrial production plant implementing the new advances in $R \& D$ and the best available Techniques (BAT). The student response has been very positive to this new methodology and it can be concluded that the use of this technology based on scientific research articles increased the students' skills within the framework of the subject.

Keywords: Cooperative learning, Integration, $R+D$, Project Real Scale

\section{INTRODUCCIÓN}

En la actualidad, la rapidez con la que están cambiando los modelos de negocio y los paradigmas empresariales obliga a las compañías a diseñar procesos productivos más flexibles, aplicar los nuevos avances en $\mathrm{I}+\mathrm{D}$ y por lo tanto se debe preparar a los alumnos para que adquieran habilidades para el futuro mejorando su capacidad de integrar los conceptos básicos con estos nuevos avances. Si bien, este hecho es patente en diversos sectores, tanto el biotecnológico como el químico son unos de los que están experimentando un mayor grado de avance e interés en la sociedad (Heitzmann et al., 2018).

El trabajo plantea una alternativa educacional para solventar el problema de la interdisciplinariedad en la educación universitaria actual. La interdisciplinariedad es altamente relevante para el sistema educacional con objeto de educar la personalidad del estudiante con pensamiento creativo y capaz de tomar decisiones responsables importantes en las condiciones de la sociedad postindustrial y aumentar la competitividad profesional en los mercados laborales locales y globales (Suhodimtseva et al., 2020) (Figura 1).

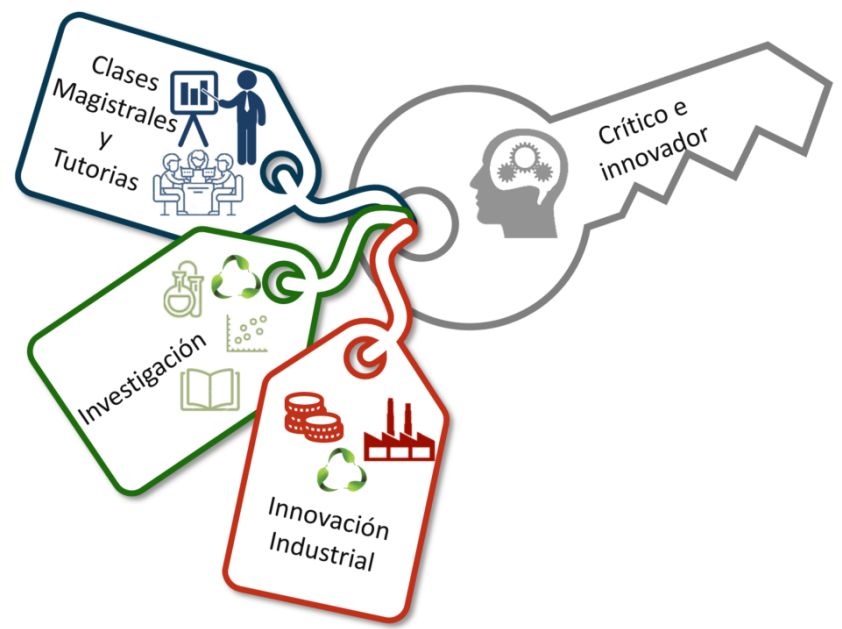

Figura 1. Interdisciplinariedad en la educación universitaria para formar estudiantes con pensamiento crítico e innovador 
Se pretende preparar a los estudiantes para el futuro teniendo en cuenta que la mejora de la competitividad de las empresas requerirá la implementación de nuevas tecnología y avances científicos, así como su adaptación medioambiental (Leong et al., 2019). Con esta idea a lo largo de estos años, en la Universidad de Vigo se ha diseñado un proyecto de innovación educativa mediante el concepto de Aprendizaje Basado en el Desarrollo de un Proceso a Escala Real (ABDPER).

ABDPER implica un estudio en profundidad de un proceso o un problema actual y buscar alternativas de mejora basadas en recientes avances de $\mathrm{I}+\mathrm{D}$, así como establecer la estrategia para su implantación en base a los conceptos adquiridos durante el año académico. Por tanto, el concepto ABDPER es un trabajo tutelado y guiado por el profesor en el que se potencia la creatividad del alumno, su capacidad de colaboración en un grupo de trabajo, así como su capacidad de implementar soluciones innovadoras en base al conocimiento de base impartido en la materia. En la Figura 2, se presenta un esquema con los principales factores que se emplean en el desarrollo de la metodología ABDPER.

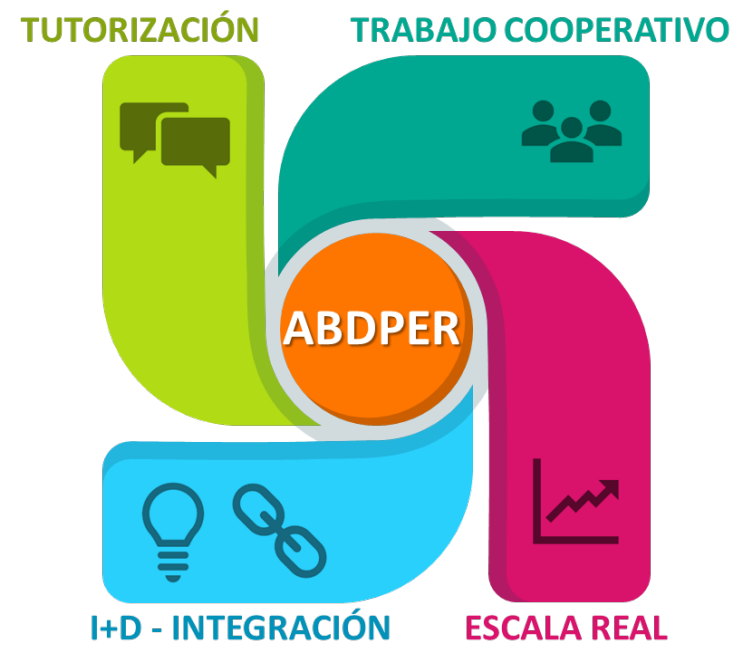

Figura 2. Factores que intervienen en la metodología ABDPER.

Si bien esta técnica de aprendizaje se ha aplicado a diversas materias de diversos grados, en este trabajo nos centraremos en la exposición de la metodología desarrollada y aplicada en una de las materias "Reactores y Biotecnología".

Con objeto de contextualizar la materia, se realizará una breve exposición de la materia y la titulación en la que está incluida. La materia pertenece al Módulo de Tecnología Específica en Química Industrial del Grado de Ingeniería en Química Industrial en la Universidad de Vigo, la cual se imparte en el segundo semestre del tercer curso de la titulación. Tal y como aparece en el plan de estudios vigente, esta materia consta de 9 créditos ECTS de carácter obligatorio. En la materia se sientan las bases de la Ingeniería de las Reacciones Químicas y los principios básicos de la Biotecnología.

En la Figura 3, se presentan los principales objetivos de esta materia, centrados en dar conocer a los estudiantes los aspectos básicos de la Ingeniería de las Reacciones Químicas, Biotecnología, así como los aspectos fundamentales del diseño de reactores para su aplicación a procesos productivos tanto en la Industria Química como en la Biotecnológica (Feijoo et al., 2018).

La Ingeniería de las Reacciones Químicas se ocupa del diseño y operación de los reactores químicos; puede decirse que es la disciplina que cuantifica la influencia de los fenómenos de transporte y la cinética, para relacionar el funcionamiento de los reactores con las condiciones y variables de entrada. Para este cometido se requieren conocimientos básicos de química, termodinámica y cinética, mecánica de fluidos y fenómenos de transporte, física, bioquímica, etc.

Por otra parte, esta asignatura sirve también para introducir al estudiante en el campo de la Biotecnología. Si bien el concepto de biotecnología ha tenido muchas definiciones, en líneas generales, la biotecnología es la tecnología basada en el empleo de sistemas biológicos y organismos vivos o sus derivados para la creación o modificación de productos o procesos para usos específicos. Por tanto, la Biotecnología integra múltiples disciplinas que contribuyen al uso inteligente de la naturaleza y los productos naturales, proporcionando procesos de producción sostenibles y respetuosos con el medio ambiente, así como nuevas herramientas que permiten avanzar con mayor precisión, garantía y rapidez en sectores fundamentales como salud, agricultura, alimentación, industria y medio ambiente.

Por tanto, se pretende proporcionar al estudiante los conceptos elementales y básicos de la materia relacionados en este caso con la Biotecnología e introducirlos en la Industria Biotecnológica actual, poniendo de manifiesto la importancia del cambio de escala y los problemas existentes con respecto al medio ambiente, la energía y los recursos naturales integrando los recientes avances en $\mathrm{I}+\mathrm{D}$ en el sector.

Finalmente, se debe destacar que en muchas de las asignaturas seleccionadas para ABDPER es el primer contacto que tienen los alumnos con la temática, como sería la biotecnología, y dependiendo de la orientación que tomen en el cuarto curso, puede ser la única vez que adquieran conocimientos sobre esta temática, por lo que se requiere que adquieran una visión general y real de los procesos y su aplicación a escala real.

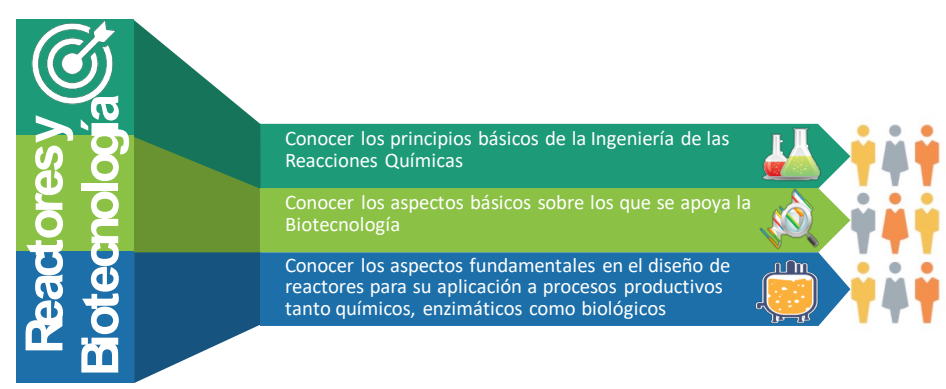

Figura 3. Objetivos principales de la materia Reactores y Biotecnología.

Como se ha comentado anteriormente, la situación actual de continua revolución científica requiere de una transformación del rol de la educación para los programas de grado y en consecuencia diseñar alguna de las asignaturas de manera que se desarrollen las habilidades de investigación de los estudiantes. En este trabajo, se presenta un estudio de caso para presentar y evaluar una nueva metodología de enseñanza / evaluación destinada a desarrollar el pensamiento crítico, la objetividad y el ingenio entre los estudiantes de grado en el sector biotecnológico, pero igualmente se está aplicando a otros 
sectores como el químico, mecánico, eléctrico,... en las diversos grados en los que se imparte materias que pueden mejorar las habilidades del estudiante en la integración de la investigación en el tejido productivo.

\section{CONTEXTO}

En el curso 2012/13, se inició la docencia de la materia "Reactores y Biotecnología", comenzando a impartirse utilizando una metodología convencional basada en la didáctica de exposición de conceptos teóricos y resolución de problemas. Sin embargo, en este primer curso se observó que los alumnos debido a la diversidad de procesos que se evalúan y su aplicación a dos sectores como el Químico y el Biotecnológico, no eran capaces de entender la aplicabilidad de la materia y de los conceptos recibido. Ante, este problema se realizó un cambio de estrategia y se introduce la realización de un trabajo tutelado con objeto de desarrollar un proceso convencional y comercial implantado de tal manera que los alumnos fueran integrando en ese trabajo todos los aspectos de interés necesarios para el diseño de una planta de producción química o biotecnológica. La experiencia fue muy positiva y los estudiantes manifestaron su aprobación de aplicar esta dinámica de estudio. No obstante, detectamos que faltaba la integración de los nuevos avances en un campo tan fructífero como el biotecnológico y por ello se inició una experiencia piloto basado en el concepto del ABDPER (Pazos et al. 2013). Posteriormente se procedió a su aplicación a otras materias.

En este caso el trabajo tutelado se propone como una herramienta apropiada para aumentar y afianzar el conocimiento del estudiante sobre los procesos biotecnológicos y que el estudiante se integre en el campo de los desarrollos de I+D y su implementación en el diseño de un proceso.

Esta metodología se basa en principios similares a los del Aprendizaje Basado en el Desarrollo de Proyecto, en la cual los estudiantes en grupos recogen y analizan información, planifican, implementan, reportan, presentan y evalúan sus proyectos (Hmelo-Silver, 2004). En concreto la metodología ABDPER, utiliza estas mismas herramientas con el objetivo de implementar los conceptos adquiridos a lo largo del desarrollo de la materia en la realización de un Proceso Industrial a Escala Real. Por tanto, esta metodología se basa en las fortalezas individuales de cada uno de los estudiantes que integran el grupo de trabajo y les permite explorar sus áreas de interés y habilidades dentro del tema propuesto. Así, se favorece el trabajo de grupos con diferentes estilos de aprendizaje, niveles de habilidades y antecedentes culturales. Los proyectos pueden incluir el diseño y desarrollo de un producto, servicio, marca, proceso, función u operación que puede ser visto o utilizado por otros, pero todos ellos de aplicación a escala real (Huet, 2018).

\section{DESCRIPCIÓN}

En las materias, incluyendo Reactores y Biotecnología, se distinguen seis metodologías docentes: Actividades introductorias, Resolución de problemas y/o ejercicios, Prácticas de laboratorio, Presentaciones/exposiciones, prácticas de ordenador, Sesión magistral y Trabajos tutelados y que son las planteadas en la memoria del título. En este trabajo se describen las modificaciones realizadas en la metodología del Trabajo tutelado. Ya que si bien inicialmente, se había diseñado esta metodología para que el alumno de manera individual realizara un trabajo, en la actualidad, se ha realizado una modificación por la cual el alumno trabajando en grupo vaya plasmando en el diseño de una planta todos los conceptos que va adquiriendo a lo largo de la materia. Por ello, los alumnos desarrollarán un trabajo en grupo relacionado con la temática de la materia que será propuesto el profesor. Posteriormente, los alumnos tendrán que realizar un estudio de los avances científicos-tecnológicos en la temática y en base a un artículo científico seleccionado deberán ir desarrollando el diseño de una planta de producción. Este trabajo se materializará en una memoria escrita, así como en la defensa pública del mismo.

\section{Resultados}

En base a lo anteriormente comentado, se ha decidido que el trabajo tutelado se focalice en el diseño y desarrollo de un proceso biotecnológico utilizando el concepto ABDPER. Es bien sabido que, como primera etapa del desarrollo de cualquier proceso industrial, son necesarios años de investigación a pequeña escala que posteriormente se traslada a planta piloto y finalmente a escala industrial. Por tanto, el objetivo es que el estudiante realice un símil del desarrollo de un proceso desde el laboratorio de $\mathrm{I}+\mathrm{D}$ al diseño de planta.

Como punto de inicio se utilizarán los artículos científicos publicados en revistas indexadas, ya que en muchos de ellos se desglosa la información básica para llevar a cabo el desarrollo de un proceso a pequeña escala. Así, la información que se les suministra a los estudiantes inicialmente es un artículo científico reciente en el cual se estudia un proceso biotecnológico a escala laboratorio/o piloto. El objetivo del proyecto es la implementación a nivel industrial del proceso biotecnológico descrito en el artículo científico (Figura 4).

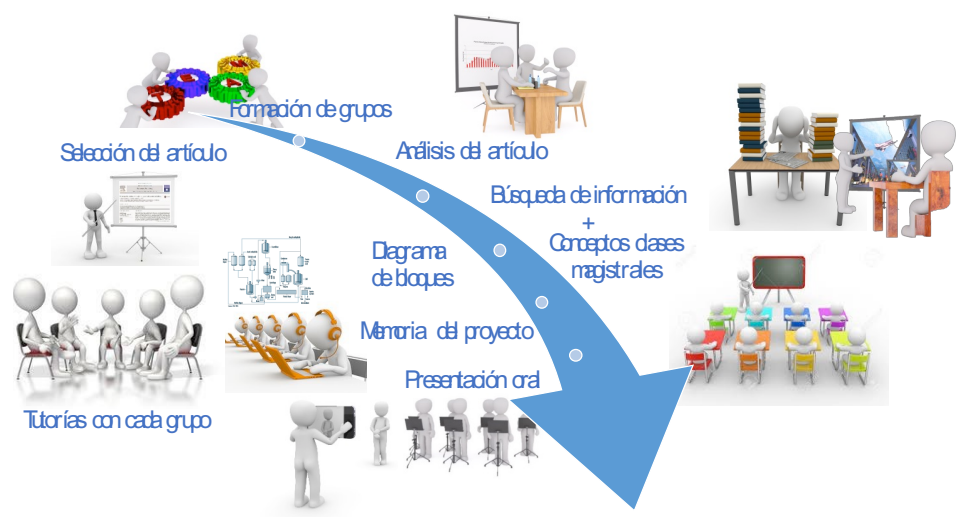

Figura 4. Esquema de la metodología ABDPER desarrollada en el trabajo tutelado.

Para ello, los estudiantes deben conocer el proceso en detalle, así como buscar las unidades operativas necesarias para el funcionamiento del proceso a escala industrial. Es por ello que los alumnos deben determinar qué conocimientos necesitan para trabajar en el proyecto, razonar y especificar un plan de acción para trabajar en el mismo. Los estudiantes se dedican al estudio individual de los materiales necesarios, búsqueda en las fuentes de información que incluyen bibliotecas, bases de datos y páginas web y que posteriormente vuelven al grupo de trabajo donde comparten la información con los compañeros, se reúnen con el profesor y trabajan en grupo para desarrollar el proyecto. De esta manera los estudiantes irán aprendiendo la realidad industrial, los problemas y las magnitudes reales de un proceso biotecnológico y obtendrán una visión integrada de los procesos. 
El proyecto debe incluir todas las etapas relacionadas con el proceso biotecnológico: propagación de cultivos (upstream), biorreactor, separación y purificación de productos (downstream). El profesor proporcionará al grupo una definición precisa del proyecto a desarrollar incluyendo los distintos hitos a alcanzar por los alumnos (entrega de los resultados parciales) y las fechas de entrega. Cada grupo debe seguir la planificación con una serie de hitos intermedios que correspondan a cada una de las entregas de las diferentes partes del proyecto. Con el fin de evaluar la memoria presentada, así como la presentación de los proyectos se propone el uso de rúbricas que se ponen a disposición de los alumnos en la plataforma TEMA para que tengan con antelación los requisitos que deben cumplir sus trabajos.

En la Figura 5, se muestra los resultados de las encuestas realizadas a los alumnos en el curso 2017/18. Como se puede observar más del $65 \%$ considera que la experiencia es muy positiva, el $33 \%$ positiva y el resto no considera que sea ni positivo ni negativo y menos del $1 \%$ considera que aplicar esta metodología ABDPER es negativa. Por tanto, en base a estos resultados positivos, se ha comenzado a aplicar a otras materias de otros grados e incluso de máster y nuevamente la valoración de los alumnos ha sido muy positiva.

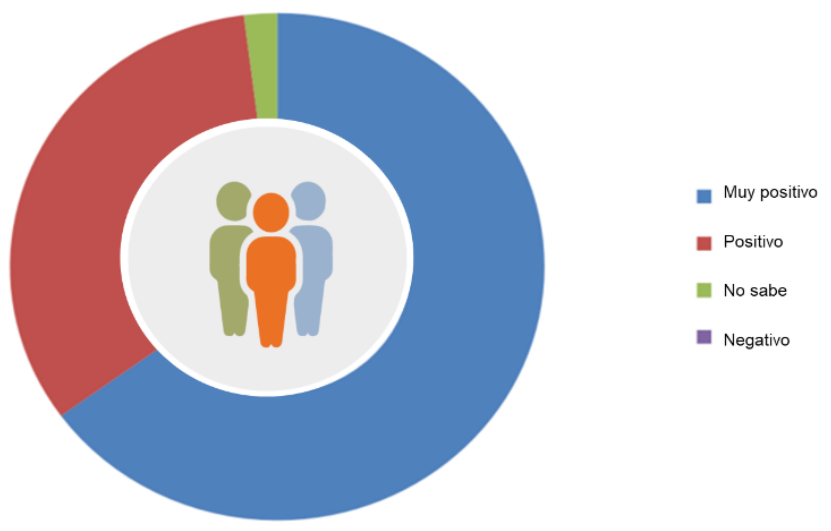

Figura 5. Grado de satisfacción de los estudiantes que han seguido la metodología ABDPER en el curso 2017/18.

\section{CONCLUSIONES}

Las principales habilidades adquiridas en el desarrollo de esta metodología son aquellas relacionadas con:

- Resolución de problemas,

- Selección y el uso de herramientas analíticas y de búsqueda que mejoran sus habilidades analíticas,

- Toma de decisiones en diferentes situaciones y

- Hacer frente a las responsabilidades y al trabajo cooperativo.

La respuesta de los estudiantes fue muy positiva a esta nueva metodología. En base a los resultados de las encuestas se puede concluir que, esta metodología, es valorada positivamente por más del 90\% de los alumnos. Además, se detecta que el conocimiento de las etapas del proceso, aplicaciones prácticas y la realidad de la biotecnología se incrementó significativamente con esta metodología aumentado las habilidades de los estudiantes en el marco de la asignatura.

\section{AGRADECIMIENTOS}

Los autores agradecen el apoyo recibido por la Universidad de VIGO y al proyecto Contrato-Programa para el desarrollo de este trabajo.

\section{REFERENCIAS}

Heitzmann, F., Clement, C., Sans, J.-P. (2018). Proposal for an innovating business model for supporting biotechnology companies, ecosystem and their founders. Journal of Commercial Biotechnology, 24(3), 6-10.

Hmelo-Silver, C.E. (2004). Problem-Based Learning: What and How Do Students Learn?. Educational Psychology Review, 16, 235-266

Huet, I. (2018). Research-based education as a model to change the teaching and learning environment in STEM disciplines. European Journal of Engineering Education, 43(5), 725-740

Feijoo, G., Ibañez, R., Herguido, J., Partal, P., Tobajas, M., Sèmpere, J., López-Pérez, M.F., Rivero, M.J. (2018). Education of chemical engineering in Spain: A global picture. Education for Chemical Engineers, 24, 27-31

Leong, W.D., Teng, S.Y., How, B.S., Ngan, S.L., Lam, H.L., Tan, C.P., Ponnambalam, S.G. (2019). Adaptive analytical approach to lean and green operations. Journal of Cleaner Production, 235, 190-209.

Pazos M., Longo M.A., Sanromán, M.A. (2013). Experiences of Innovation Teaching in Bioprocess Engineering University Course. Romanian Journal for Multidimensional Education, 5(1), 123-139.

Suhodimtseva, A.P., Vorozheikina, N.I., Eremina, J.B. (2020). Integration approach to solving problems of interdisciplinary nature in the conditions of postindustrial education. Smart Innovation, Systems and Technologies, 138, 501-510.

Wei, P., Cotton, D., Murray, P. (2014). Linking Research and Teaching: Context, Conflict and Complementarity. Innovations in Education and Teaching International, 51(1), 3-14 